\title{
Radiofrequency Ablation of Renal Tumors
}

\author{
Michael J. Stone, MD [Research Fellow] ${ }^{\star}$, Aradhana M. Venkatesan, MD [Staff Clinician] $]^{\dagger}$, \\ Julia Locklin, RN, MS, CCRC [Research Nurse Specialist] ${ }^{\ddagger}$, Peter Pinto, MD $\S$, Marston \\ Linehan, MD\$, and Bradford J. Wood, MD [Chief] ${ }^{\top}$ \\ *National Institutes of Health Clinical Center, Diagnostic Radiology Department, Bethesda, Md. \\ $\uparrow$ National Institutes of Health Clinical Center, Diagnostic Radiology Department, Bethesda, MD. \\ $\$$ National Institutes of Health Clinical Center, Diagnostic Radiology Department, Bethesda, MD. \\ §Urologic Oncology Branch, National Cancer Institute, Bethesda, MD. \\ TInterventional Radiology, Diagnostic Radiology Department, National Institutes of Health Clinical Center, \\ Diagnostic Radiology Department, Bethesda, MD.
}

\begin{abstract}
Radiofrequency ablation (RFA) of renal tumors is a promising technique that plays a unique and increasingly important role in urologic oncology practices. RFA is appealing as a minimally invasive therapy that may be performed on an outpatient basis. It enables treatment of an area 3 to $5 \mathrm{~cm}$ in diameter, with relatively low morbidity and mortality rates. Most interventional radiologists (IRs) are familiar with RFA of liver tumors, and several principles and techniques used in the liver may be extrapolated for use in the kidney. However, it is crucial to bear in mind that local tumor ablation in the kidney presents unique challenges, secondary to the kidney's unique anatomic and physiologic features. Clinical and technical considerations, risks, and complications pertaining to RFA of renal tumors are reviewed here, including approaches commonly used in our practice.
\end{abstract}

\section{Keywords}

radiofrequency ablation; kidney; renal cell carcinoma; Von Hippel-Lindau syndrome

Renal cell carcinoma (RCC) continues to be a significant source of morbidity and mortality despite advances in minimally invasive surgical and percutaneous options. RCC accounts for approximately $85 \%$ of renal tumors. ${ }^{1,2}$ Most interventional radiologists (IRs) are familiar with radiofrequency ablation (RFA) of liver tumors, but local tumor ablation in the kidney presents unique challenges.

Recently, renal tumor RFA has become an invaluable tool for the IR. Initially, RFA of renal tumors began in patients who were nonoperative candidates since the "gold standard" treatment for RCC of any size had historically been radical nephrectomy. With the advent of nephron sparing surgery, renal tumor removal with prevention of the surrounding kidney is now often the first choice in RCC management. Some would argue that this should no longer be the case, as RFA has proven to be a benefit in patients with a history of prior nephrectomy or solitary kidney, hereditary or familial renal cancer, and baseline renal insufficiency. In addition, nonstandard indications for renal RFA have been described and now include transfusion-

Address reprint requests to: Bradford J. Wood, MD, Diagnostic RadiologyDepartment, National Institutes of Health Clinical Center, 1N-264, Department of Diagnostic Radiology, 10 Center Drive MSC 1182, Bethesda, MD 20892. E-mail: BWood@cc.nih.gov.. 
dependent hematuria, pain control, palliation, preoperative tumor embolization, postbiopsy bleeding, and transitional cell carcinoma. RFA may also aid in preserving lifetime renal function in patients with hereditary syndromes having a propensity for renal malignancies, such as Von Hippel-Lindau syndrome, in whom multiple renal neoplasms and recurrence over time are likely. RFA can also benefit the 3 to $5 \%$ of patients with sporadic bilateral RCC. ${ }^{3,4}$ Given the increased importance of percutaneous RFA in the treatment of renal tumors, this article reviews the clinical and technical considerations needed to perform RFA in this organ system, the associated risks and complications, and the approaches commonly used in our practice.

\section{Treatment Planning}

In our practice, treatment planning begins with a multidisciplinary evaluation involving urologists, medical oncologists, interventional radiologists, and radiation oncologists. This multidisciplinary team is well suited to evaluate the medical history and preprocedural imaging, inform the patient, and guide them through the various treatment options. Risk assessment and treatment goals must be defined before selection of RFA as a treatment choice.

Indications for RFA are influenced by lesion size, location, comorbidities, and patient preferences. Patients who are not candidates or do not choose RCC removal often fall into one of two distinct categories. One subset of patients are those with a solitary tumor, measuring less than 3 to $4 \mathrm{~cm}$ in the greatest dimension, and normally functioning native kidneys. These are usually patients with sporadic RCC. In these patients, the physician may be more aggressive with an intent to cure and, therefore, should plan for a wide ablation margin. In this patient population the greatest feared risk is local recurrence, as the patient has ample normal renal tissue to allow for a wide ablation margin. The second discrete patient group consists of patients with a hereditary syndrome, such as Von Hippel-Lindau, which predisposes to multiple, recurrent clear cell renal neoplasms over their lifetime. In these patients, renal failure is a future risk, regardless of the ablation procedure, and this risk can be hastened by overly aggressive treatment with RFA. Patients who have previously undergone contralateral nephrectomy or partial nephrectomy are similarly at risk for renal failure. It is essential in these patients to verify baseline renal function before treatment. In our practice, a 24-hour urine collection is used before and after ablation to compare creatinine clearance. A nuclear medicine renal study can also be useful in following renal function or the laterality of renal function percentages, before and after ablation. A smaller, less aggressive ablation zone is also preferable in these patients, with a less than 5- to 10-mm margin of normal parenchyma, depending on renal reserve and concomitant disease. Tumor size is also a consideration for patient selection. Previous research has indicated that smaller tumors provide a greater chance for local ablation success, with the greatest success in tumors less than 3 to $4 \mathrm{~cm} .{ }^{5}$ Even larger tumors have been treated; however, risk profiles and goals (palliation, debulking) should be clearly defined at the outset.

\section{Technical Considerations}

Procedural planning begins with a review of prior imaging to determine the optimal electrode path. Renal tumors are most readily accessible via a posterior or lateral approach with the patient in a semiprone or lateral decubitus position. This helps minimize the risk of bowel injury, as bowel loops are more likely to shift into the treatment path with the patient in the supine position. Clear communication with the anesthesiologist or the patient, if possible, to coordinate breathing can help avoid transgression of the pleura, or enable movement of a target lesion off of an intervening rib. Multiple scans and repositioning of the electrode (and/or patient) may be necessary to achieve the desired probe-tumor relationship. Hydrodissection, described herein, may also be used at this stage of the procedure for protection of surrounding 
tissues. Increasing anesthetic dose immediately before the onset of ablation provides the optimal combination of patient comfort and safety.

The anatomic location of the kidney must be considered. As with treatment of hepatic lesions, treatment in the kidney puts the surrounding organs and tissues at risk for thermal injury. The ureters, surrounding bowel, and several nerves and muscle are at high risk for thermal injury. The genitofemoral and ilioinguinal nerves both course along the psoas muscle and their injury can lead to unwanted groin parasthesias and diminished leg extension. The proximity of the adrenal gland to the kidney must also be taken into consideration, as damage to the adrenal gland during renal ablation can lead to an acute hypertensive crisis. The IR must be aware of this potential complication when the ablation zone abuts the adrenal gland. Damage to the adrenal gland can also result in adrenal insufficiency in certain patient populations, typically those with a history of prior nephrectomy with unilateral adrenalectomy. One must also consider the patient's past surgical history. Prior intraabdominal surgeries predispose the patient to adhesions. These adhesions can result in distortion of bowel loops and thereby pose challenges for probe placement.

Renal tumors' increased propensity for cystic elements also adds complexity to probe selection and placement. When treating a mixed cystic lesion with high malignant potential (eg, Bosniak III and IV lesions), the probe must be moved multiple times to ablate the solid components for complete treatment. Hereditary cystic renal tumors often fill in with solid components, and it may be prudent to wait to ablate a cystic neoplasm until more than half the lesion is solid, although this is speculative.

Treatment success is also influenced by tumor location. Exophytic lesions are often more readily ablated due to the presence of surrounding fat, whose presence not only makes lesions easier to target and monitor but which may also provide an insulating "oven effect" during ablation. Cortical renal tumors are also more readily ablated compared with medullary lesions. Medullary tumors suffer from an increased heat sink effect due to increased vascularity, although we have experienced a very high rate of successful ablation of medullary tumors. Therefore, a medullary tumor location should not necessarily be a contraindication to ablative therapies, but should be taken into account when planning treatment parameters and risk profiles, since medullary location predisposes to risks of urinoma, fistula, infarct, or kidney damage.

The use of landmarks for lesion localization is advisable. Common useful landmarks include nearby renal cysts, blood vessels, or the patient's own characteristic renal architecture (eg, renal fat, lobation, pyramids, etc). Small masses may be transiently conspicuous on magnetic resonance (MR) or enhanced computed tomography (CT). It is also not uncommon for hereditary tumors to be difficult to localize or identify, when located adjacent to numerous confounding hereditary lesions and seen only briefly following a bolus during enhanced CT or MR. In some instances, small contrast boluses $(30-70 \mathrm{~mL})$ before ablation have been used successfully to increase lesion conspicuity (Fig. 1) or after therapy to evaluate the size of the ablation zone (Fig. 2). Mini-boluses may also be used immediately postablation to compare the treatment zone to the pretreatment tumor location. In patients with a history of iodinated contrast allergy, premedication may be performed to enable contrast administration.

In the past, many renal lesions felt to be inaccessible for percutaneous ablation were triaged for laparoscopic ablation. Today, almost all renal masses are approachable percutaneously following hydrodissection, with the exception of some anterior lesions that lack a safe trajectory. ${ }^{6}$ Using ultrasound (US) guidance, a 22-G Chiba needle (Cook Medical, Bloomington, IN) is inserted into the desired potential space and a small volume $(\sim 50 \mathrm{~mL})$ of $5 \%$ dextrose in water (D5W) is injected. As normal saline can conduct electricity, it is not 
recommended for hydrodissection. ${ }^{7}$ Needle placement is confirmed with CT or US. Next, a side hole needle-sheath catheter (Yueh, Cook Medical, Bloomington, IN, or Skater Centesis Catheter, Angiotech, Vancouver, BC) is inserted, its stylet removed, and D5W hung to gravity with a stopcock in series. Free-flowing fluid indicates appropriate intra- or retroperitoneal placement, and the desired volume can then be allowed to infuse. The total volume instilled depends on the amount of organ displacement needed to avoid heating nontarget tissue. High volumes of infusion may result in significant hyponatremia, which can be avoided with the use of preprocedural IV normal saline. Sequential CT imaging confirms displacement of the regional anatomy. Hydrodissection is most useful in protecting bowel, psoas muscle, ureter, and adjacent nerves (Figs. 1 and 3). If the initial D5W infusion does not move or adequately protect the anatomy of concern, the IR may attempt repositioning the patient to encourage the proper alignment. It is important to bear in mind, however, that in some patients, the presence of intraabdominal adhesions may prevent successful hydrodissection. The ureter is particularly at risk for thermal damage with centrally located neoplasms, which warrants special care in these instances. In such cases, use of a retrograde chilled infusion via ureteral catheter or antegrade infusion via nephrostomy may be performed. ${ }^{8}$ Such procedures are often performed in conjunction with a urologist, who can perform cystoscopy and cannulate the ureters retrograde.

Electromagnetic (EM) tracking (Fig. 4) is a novel navigation tool that can further facilitate needle placement (Percunav; Traxtal Inc, Toronto, ON, and Philips Medical Systems, Andover, MA). With this technology, fiducial skin markers are placed at the time of preprocedural CT or MR, or at the beginning of the procedure. The ablation navigation system then allows multiple preprocedural imaging modalities to be used with real-time feedback during the RFA, showing the location of the electrode and treatment zone in relation to the enhanced CT or MR target. Both ultrasound (Fig. 5) and CT are always used for renal RFA in our practice; coregistration of these images, or those of other modalities, is enabled via a commercial system for EM tracking (Traxtal Inc, Toronto, ON).

As in hepatic RFA, it is generally optimal to follow a "deep first" approach when planning sequential ablation zones. In both the liver and the kidney, this helps minimize the effect of generated gas on ultrasound image quality. However, it is paramount to recognize that not all of the rules for ablation of tumors in the liver hold true for the kidney. For example, infarcts are more likely to occur following renal RFA than with hepatic RFA, given the same energy and treatment time. Infarction may result in greater organ damage than anticipated, which can be a significant complication if future therapy is intended, as in patients with Von HippelLindau syndrome. The kidney is an end organ in terms of blood supply, while the liver possesses a dual blood supply. This lack of a dual blood supply may be exploited in renal RFA, as the deep first approach has the advantage of potentially eliminating or diminishing blood flow to more peripheral ablation zones. This is a technical advantage that cannot usually be exploited in the liver, given its dual blood supply.

The unique physiologic environment of the kidney may also result in a smaller ablation zone than one would expect from liver RFA. The kidney has 5 times greater blood flow per gram than the liver, which can lead to a more significant heat sink effect from global or local blood circulation. ${ }^{9}$ The $3-\mathrm{mm}$ rule that governs "significant" vessel size for heat sink considerations in the liver ${ }^{10}$ may therefore not apply to the kidney. With an increased risk of heat sink, postRFA temperatures become a more important consideration. Attainment of a greater than $70^{\circ}$ C post-RFA temperature (ie, with the Valleylab Cool-tip system) may be attainable only after a double treatment at the same location. Greater importance is therefore also placed on overlapping ablation zones in the kidney, as well as repeating treatments at the same or adjacent locations. 
Given these unique physiologic parameters, the IR must maintain a high level of suspicion for undertreatment of renal tumors. There are technical cues that undertreatment may have occurred, many of which are specific to the RFA system in use. With the Valley Lab system (Boulder, CO), a post-RFA temperature maximum of less than $70^{\circ} \mathrm{C}$, rapid decline in temperature during cooling (indicating persistent cooling perfusion at the tip), or a lack of pulsing of the generator may all indicate insufficient treatment and the need for a repeat ablation sequence, or greater anatomic overlap of successive treatments. With the Boston Scientific system (Natick, MA), a lack of rise in impedance or a delayed "roll off" may similarly indicate suboptimal treatment. With the Angiodynamics/RITA system (Queensbury, NY), consistently low temperatures throughout the procedure point toward undertreatment. IRs should be familiar with the parameters and tendencies of the system employed in their practice to assess need for repeat treatments or tighter overlapping regions.

Additional risks and complications of renal RFA include infection, tumor seeding, and hemorrhage. As such, it is also our practice to use antibiotic prophylaxis with a fluoroquinolone. Abscess formation has been reported postablation and can even mimic tumor recurrence. ${ }^{11}$ Tumor seeding along the probe track may follow tumor biopsy or ablation; its likelihood is minimized by ablating the probe track during removal. While hemorrhage is a potential complication, its likelihood is significantly lower with RFA compared with nephrectomy, given the cauterization and coagulation necrosis inherent to the technology. This feature has been exploited in the use of renal RFA to treat life-threatening, transfusion-dependent hematuria (Fig. 6) and also for hemorrhage after partial nephrectomy. ${ }^{12}$

The method of ablation is also a decision for the IR to make when planning treatment. Cryoablation of renal tumors is an alternative option to RFA, and will be discussed in detail elsewhere in this issue. There are anecdotal reports that cryoablation may provide increased protection to the urothelium in those tumors having broad contact with the collecting system. 13 However, the clinical validity and relevance of these observations remains unproven in human practice. If true, this protection would be expected to result in fewer urinomas and urinary fistulas in cryoablation patients. Although some IRs feel that the "ice ball" generated during cryoablation is more readily visualized with ultrasound compared with the burn that is the result of RFA, both RFA and cryoablation are limited by the generation of gas and ice, respectively, which impede sonographic visualization. Some investigators contend that the proximal zone of ablation is better visualized with cryoablation compared with RFA. However, the visualized edge does not precisely correlate with the zone of ablation for either cryoablative or RFA techniques, and some investigators contend that ultrasound signal-to-noise ratio is better at all edges of the burn for RFA than cryoablation.

\section{Follow-Up}

Critical to the success of RFA is the use of a standard follow-up algorithm. The timing of follow-up imaging intervals may be similar to those used after liver RFA. MR imaging may be safer than contrast-enhanced $\mathrm{CT}$ for those patients with an adequate glomerular filtration rate (GFR) to tolerate gadolinium. Immediate postablation or periprocedural imaging, or imaging within the first 1 to 3 days posttreatment, is not uncommon; however, this imaging time point is often omitted in favor of initial posttreatment imaging from 1 to 6 weeks following treatment. Many investigators perform follow-up imaging again at 3 months posttreatment and every 3 months for 1 year. Most recurrences or residual tumors are visible in the first 3 months; however, we have observed remote tumor recurrences many months beyond this time especially with slow-growing hereditary renal cell carcinomas. Conversely, a given tumor may change growth rates in patients with hereditary renal tumors making ongoing surveillance crucial. After a year, future follow-up is disease dependent. The IR must assess the level of suspicion for recurrence. Some IRs perform imaging follow-up every 6 months for the second 
year and then yearly thereafter, assuming no new lesions develop in the interval. Factors influencing the frequency of follow-up imaging include whether the treated tumor was sporadic or a result of a hereditary syndrome, and whether there was initial tumor undertreatment that required repeat treatment.

\section{Conclusion}

RFA of renal tumors is a promising technique that plays a unique and increasingly important role in many urologic oncology practices. While several ablation techniques used in other organs are applicable in this setting, the kidney is a unique organ with very important technical idiosyncrasies. Attention to these factors will maximize the success rate of the IR in using this technology for the nonoperative treatment of renal malignancies.

\section{Acknowledgments}

We thank Jochen Krücker, PhD, Philips Research North America, Briarcliff Manor, NY, and Sheng Xu, PhD, Philips Research North America, Briarcliff Manor, NY, for their assistance in the generation and compilation of the images for this article.

This work was supported in part by the Intramural Research Program of the National Institutes of Health.

\section{References}

1. Laber DA. Risk factors, classification, and staging of renal cell cancer. Med Oncol 2006;23:443-454. [PubMed: 17303902]

2. Lipworth L, Tarone RE, McLaughlin JK. The epidemiology of renal cell carcinoma. J Urol 2006;176:2353-2358. [PubMed: 17085101]

3. Grimaldi G, Reuter V, Russo P. Bilateral non-familial renal cell carcinoma. Ann Surg Oncol 1998;5:548-552. [PubMed: 9754765]

4. Siemer S, Uder M, Zell A, et al. Bilateral kidney tumor. Therapy management and histopathological results with long-term follow-up of 66 patients. Urologe A 2001;40:114-120. [PubMed: 11315585]

5. Gervais DA, McGovern FJ, Arellano RS. Radiofrequency ablation of renal cell carcinoma: part 1. Indications, results, and role in patient management over a 6-year period and ablation of 100 tumors. AJR Am J Roentgenol 2005;185:64-71. [PubMed: 15972400]

6. Lee SJ, Choyke LT, Locklin JK. Use of hydrodissection to prevent nerve and muscular damage during radiofrequency ablation of kidney tumors. J Vasc Interv Radiol 2006;17:1967-1969. [PubMed: 17185695]

7. Laeseke PF, Sampson LA, Winter TC III. Use of dextrose 5\% in water instead of saline to protect against inadvertent radiofrequency injuries. AJR Am J Roentgenol 2005;184:1026-1027. [PubMed: 15728639]

8. Schultze D, Morris CS, Bhave AD. Radiofrequency ablation of renal transitional cell carcinoma with protective cold saline infusion. J Vasc Interv Radiol 2003;14:489-492. [PubMed: 12682207]

9. Berne, RM.; Levy, MN., editors. Physiology. 4th ed.. Mosby; St. Louis, MO: 2007.

10. Lu DS, Raman SS, Vodopich DJ. Effect of vessel size on creation of hepatic radiofrequency lesions in pigs: assessment of the "heat sink" effect. AJR Am J Roentgenol 2002;178:47-51. [PubMed: 11756085]

11. Roarke MC, Collins JM, Nguyen BD. Indolent enterococcal abscess mimicking recurrent renal cell carcinoma on MR imaging and PET/CT after radiofrequency ablation. J Vasc Interv Radiol 2006;17:1851-1854. [PubMed: 17142718]

12. Wood BJ, Grippo J, Pavlovich CP. Percutaneous radio frequency ablation for hematuria. J Urol 2001;166:2303-2304. [PubMed: 11696761]

13. Janzen NK, Perry KT, Han KR. The effects of intentional cryoablation and radio frequency ablation of renal tissue involving the collecting system in a porcine model. J Urol 2005;173:1368-1374. [PubMed: 15758807] 

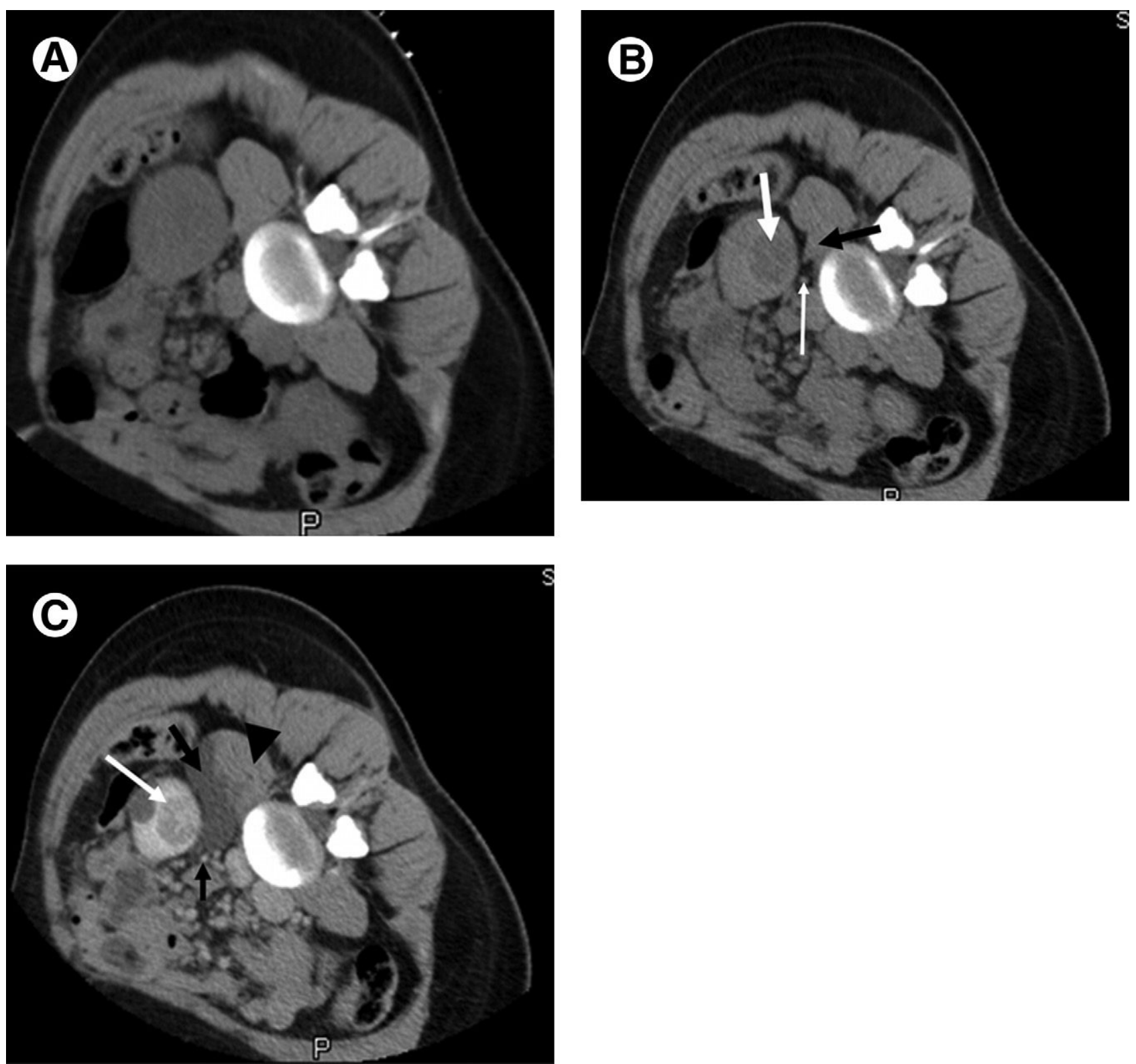

Figure 1.

Use of intravenous contrast during RFA procedure to increase the conspicuity of the lesion and other important structures. (A) Unenhanced axial CT showing right kidney before RFA; the patient's right lower pole lesion is not well seen. (B) Following a mini-bolus of $40 \mathrm{~mL}$ of IsovueM-300, the lesion is more conspicuous (thick white arrow) and noted to be adjacent to the right psoas muscle (black arrow) and ureter (thin white arrow). (C) Subsequent hydrodissection with $115 \mathrm{~mL}$ of D5W (large black arrow) and repeat bolus with $75 \mathrm{~mL}$ of Isovue-M-300 enables safe and accurate visualization and ablation of the target lesion (white arrow), which is separated from the ureter (small black arrow) and the psoas muscle (black arrowhead). 

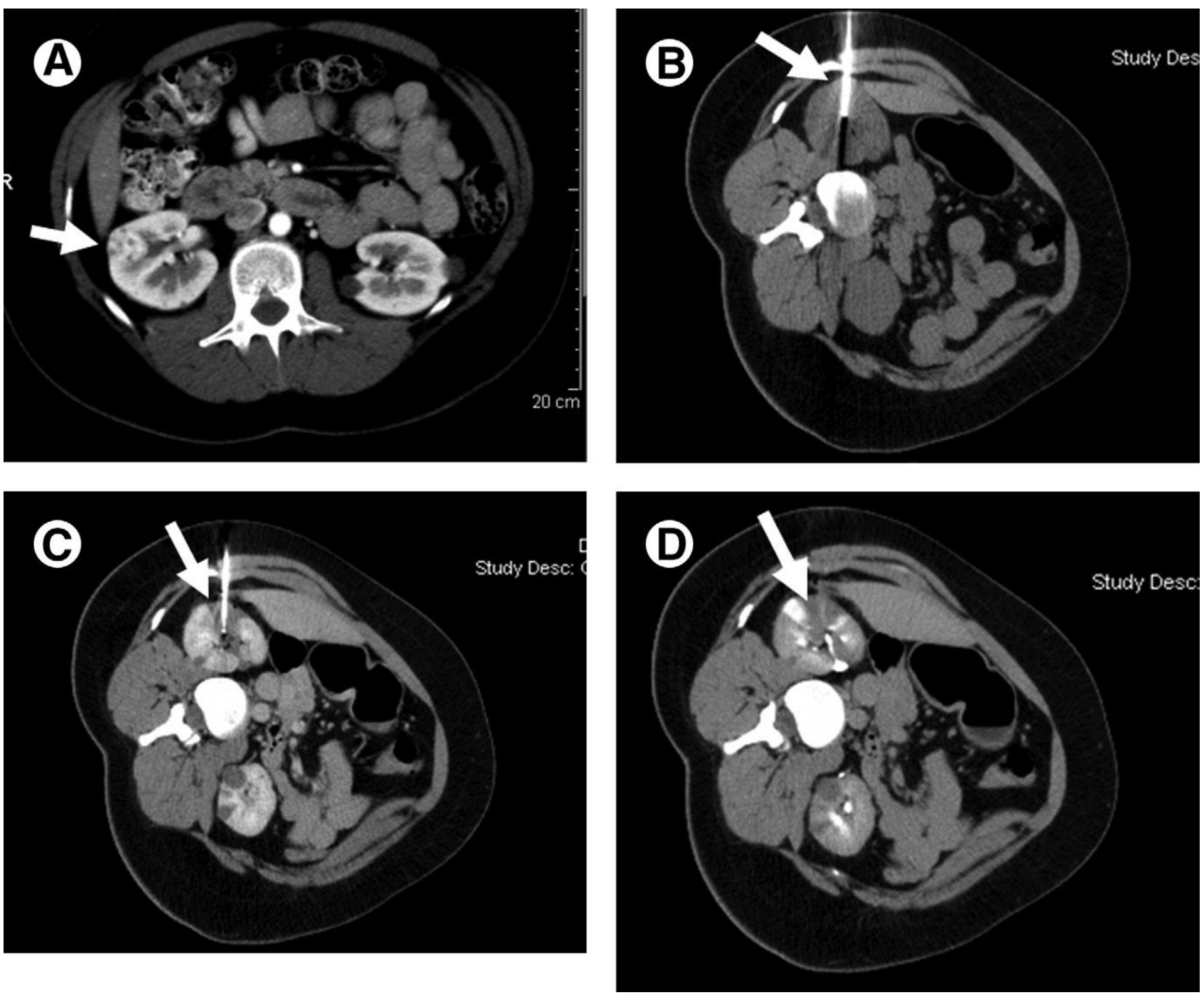

Figure 2.

RFA of a kidney tumor in a patient with Von Hippel-Lindau syndrome. (A) Preprocedure contrast-enhanced CT demonstrates a right upper pole renal cell carcinoma (white arrow). (B) RFA electrode placed into the lesion (white arrow). (C and D) Postablation contrast-enhanced CTs during the corticomedullary (C) and excretory phases (D) demonstrate the zone of ablation (white arrow), which corresponds to the target lesion. 

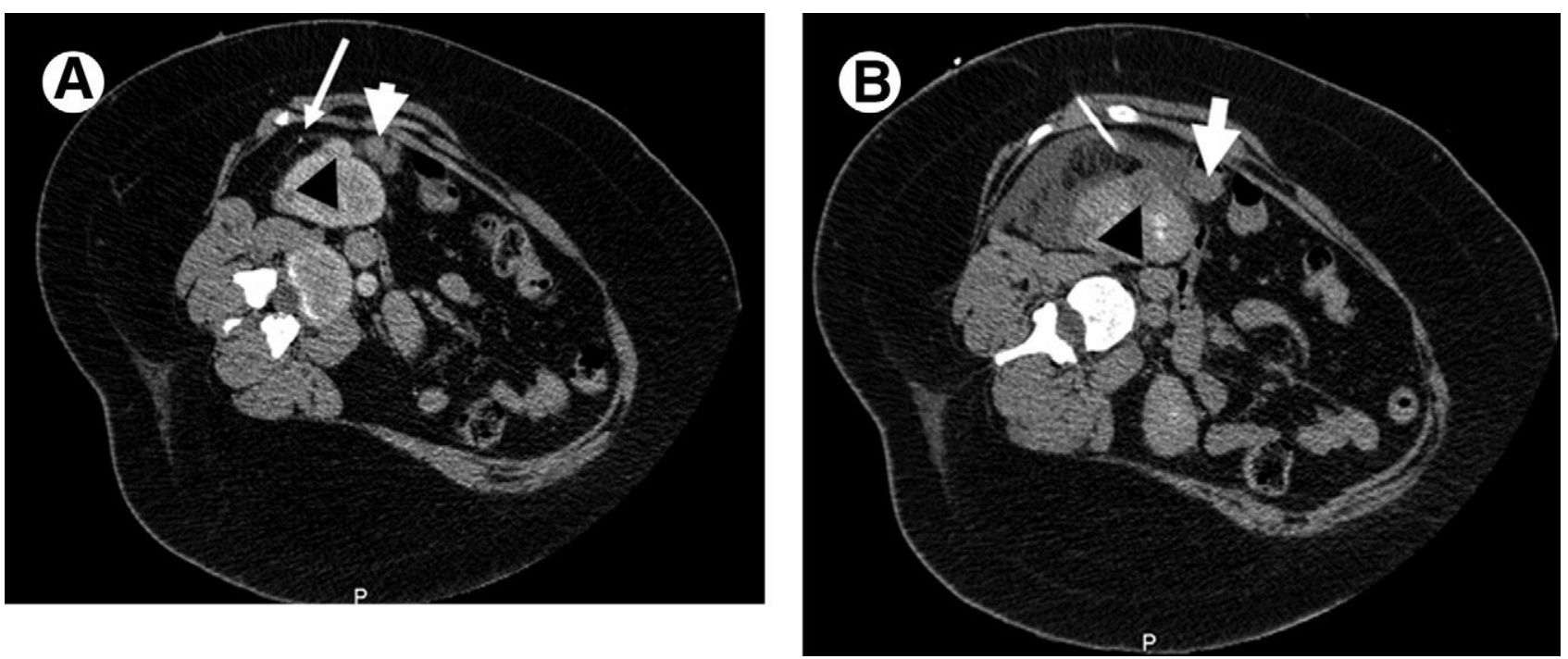

Figure 3.

Use of hydrodissection technique during RFA of a kidney tumor to avoid bowel and mesenteric arterial injury. (A) Contrast-enhanced CT demonstrating the target right renal neoplasm (black arrow) adjacent to a loop of bowel (thick white arrow) and mesenteric vessel (thin white arrow). (B) Hydrodissection with $260 \mathrm{~mL}$ of D5W displaces bowel (thick white arrow) and mesenteric vessel (not well seen) away from the target lesion, enabling safe ablation. 

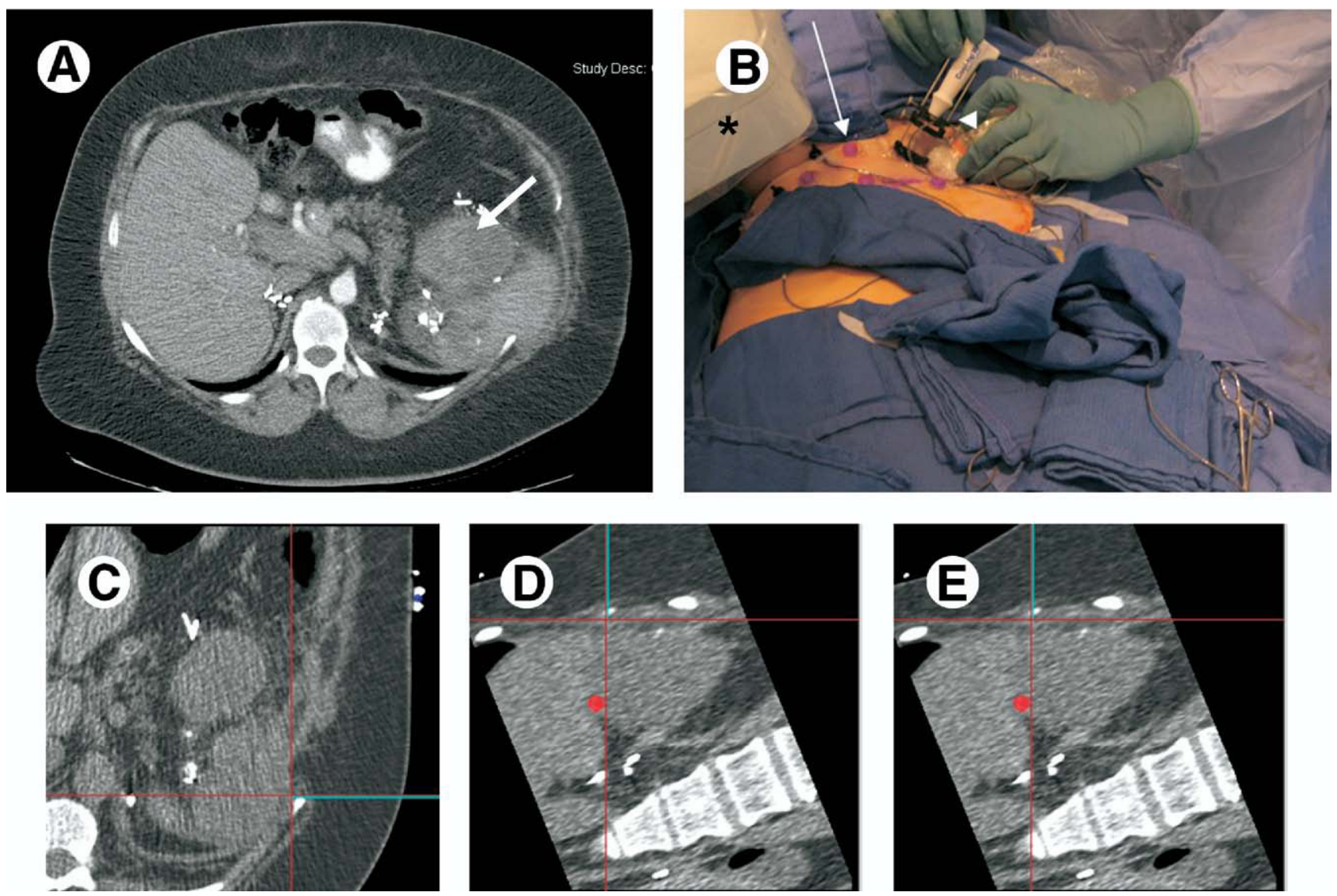

Figure 4.

Electromagnetic tracking guides renal tumor RFA. (A) Preprocedure contrast-enhanced axial CT demonstrates an exophytic left upper renal neoplasm. (B) Fiducial markers (long white arrow) on the patient's skin surface enable registration of the coordinates of an electromagnetic field with those on corresponding CT images, allowing for real-time tracking of the RFA electrode guide. (Black asterisk = electromagnetic field generator; white arrowhead = tracked electrode guide). (C-E) Real-time 3-dimensional visualization and navigation during RFA is made possible by tracking the tip of the guiding instrument using tiny sensors embedded in the electrode guide. Images from the graphic user interface demonstrating coregistered data, enabling real-time display of instrument position and orientation. The cross hairs generated by electromagnetic needle tracking are fused with multiplanar reconstructed CT images; the location of the target lesion is marked with the red dot. As the needle tip approaches the target, the red circle is seen to converge onto the red dot. (Color version of figure is available online.) 

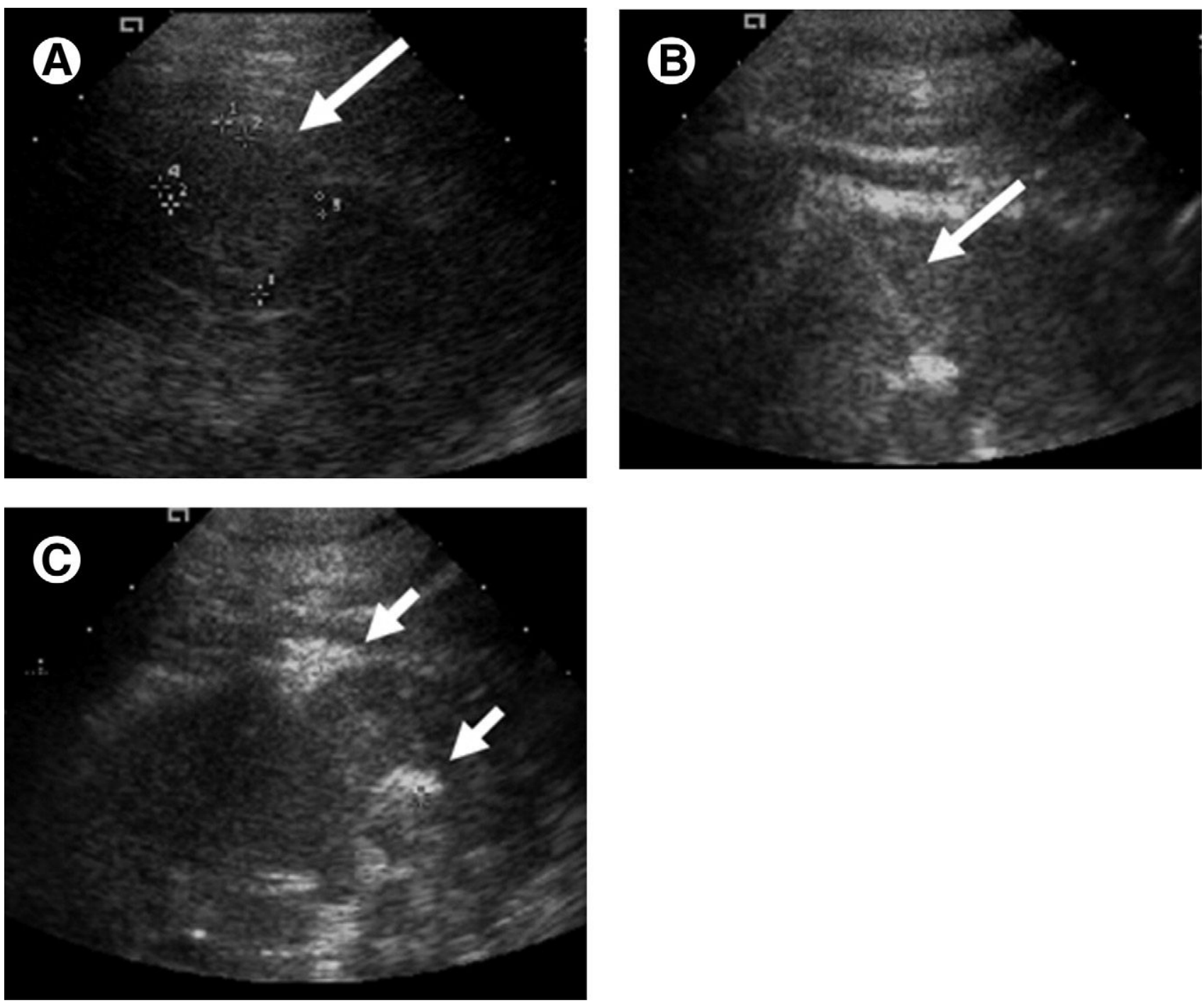

Figure 5.

Ultrasound guidance for renal RFA. (A) Ultrasound of the right kidney demonstrates a hyperechoic renal mass (white arrow). (B) Initial needle placement within the mass is confirmed sono-graphically (white arrow). (C) Subsequent ablation generates hyperechoic gas along the proximal and distal aspects of the electrode, flanking the mass (paired white arrows). 

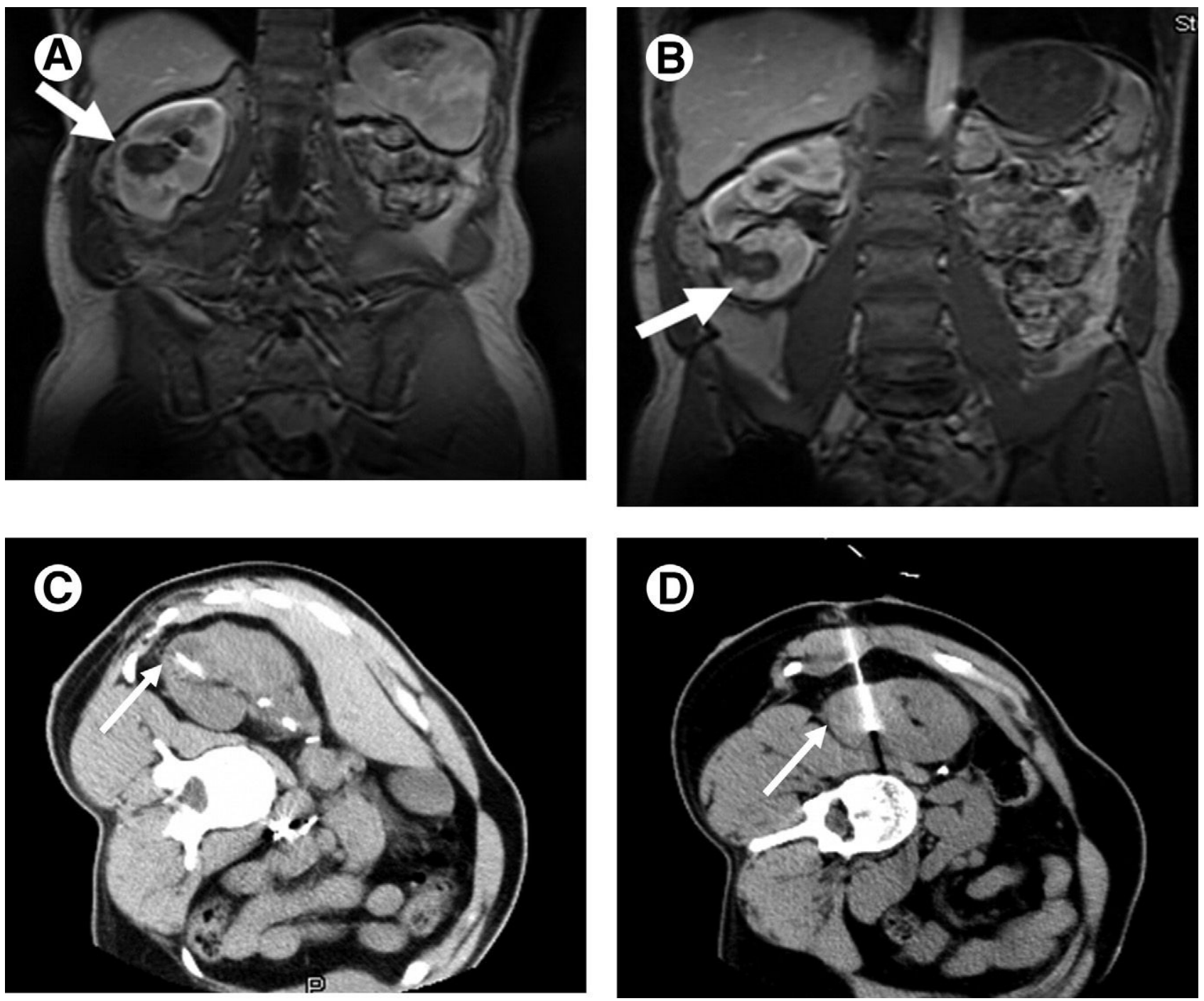

Figure 6.

Multifocal cystic renal cell carcinoma causing refractory hematuria. (A and B) Coronal postgadolinium MR imaging demonstrates 2 cystic right renal masses abutting the renal collecting system, within the midportion and right lower pole, respectively (white arrow). (C and D) RFA of both the right mid (C, black arrow) and right lower pole renal neoplasms (D, black arrow) successfully resolved the patient's hematuria. 